\title{
1. Introduction to Classroom as Organization
}

In this book, the authors describe the Classroom as Organization (CAO) teaching method, a highly experiential approach to classroom structure and management. They provide readers with information about the background of the method, considerations for its use, implementation ideas and resources for additional reading.

Chapter 2 begins with an overview of the historical context in which $\mathrm{CAO}$ emerged, and the teaching philosophy of educational constructivism that provides the theoretical basis for core teaching strategies in CAO. In addition, the chapter includes a synthesis of the CAO literature: identifying seminal articles, tracing the expansion of the methodology, and identifying themes specific to the $\mathrm{CAO}$ framework. Recognizing the diversity of both published and unpublished CAO designs, the authors conclude the chapter with a discussion of common CAO elements, including interdependence, peer assessment, student learning and management roles, instructor delegation as a senior manager, and the balance of structure and ambiguity to support learning.

In Chapter 3, the authors introduce key considerations for using $\mathrm{CAO}$ by describing the inner workings of the $\mathrm{CAO}$ methodology and assessing its utility for a given course. The chapter focuses on two sets of key considerations when contemplating the use of CAO. The first considerations address whether $\mathrm{CAO}$ is a good fit with the educator's teaching philosophy, the institutional context, the basic parameters of the course, and the cognitive and affective learning objectives. The second set of considerations relate to design elements specific to the CAO approach, including organizational design, team descriptions, peer teaching, peer assessment, assessment structures, and end-of-semester evaluation. For advanced experiential learning instructors, the authors discuss considerations for tailoring $\mathrm{CAO}$ instructional designs.

Chapter 4 contains a complete CAO course design for an upper-level organizational behavior course, OB Inc., explained in the context of the 
considerations discussed in Chapter 3. A set of appendices are provided that enable educators to adopt and adapt the design.

In Chapter 5, the authors provide an annotated bibliography of forty seminal articles in the $\mathrm{CAO}$ literature. While $\mathrm{CAO}$ sits within the broader domain of experience-based learning, the articles included in the bibliography focus directly on CAO. The bibliography is structured in chronological order so the reader can see the earliest approaches to CAO design along with later adaptations to entrepreneurship, leadership, and introduction to business courses. 